\title{
A method for rapid detection of rifampicin-resistant isolates of Mycobacterium leprae
}

\author{
NADINE HONORÉ*, PAUL W. ROCHE**, \\ JACQUES H. GROSSET*** \& STEWART T. COLE* \\ *Unité de Génétique Moléculaire Bactérienne, Institut Pasteur, \\ 28 Rue du Dr Roux, 75724 Paris, Cedex 15, France \\ **Mycobacterial Research Laboratory, The Leprosy Mission, \\ Anandaban Leprosy Hospital, PO Box 151, Kathmandu, Nepal \\ ***SAMU Social de Paris, 35 Avenue Courteline, 75012 Paris, \\ France
}

Summary A genotypic method for predicting rifampicin resistance in Mycobacterium leprae has been developed and rigorously tested on mouse footpad-derived and clinical specimens. A series of immobilized oligonucleotide capture probes can discriminate between wild type and mutant $r p o B$ alleles, and positive controls are available for the most frequent mutation affecting Ser425. Two different nonradioactive detection formats have been tested with comparable success in both an industrialized and a developing country. The standardized procedure could now be used in a prospective study of potential rifampicin resistance among multibacillary patients.

\section{Introduction}

Rifampicin is the backbone of the multidrug therapy currently used to treat leprosy ${ }^{1,2}$ and it, or a related rifamycin, will almost certainly be included in any new regimens that may be developed. ${ }^{3}$ Fortunately, resistance to rifampicin is rare and has only been documented in a limited number of lepromatous patients, mostly receiving monotherapy or other inadequate treatment. ${ }^{4}$ On characterization, all rifampicin resistant isolates of Mycobacterium leprae were found to harbour mis-sense mutations in a short segment of the $r p o B$ gene, encoding the $\beta$-subunit of DNA-dependent RNA polymerase. ${ }^{5-8}$ The same region, termed RIF-RDR has been shown to be associated with rifampicin resistance in almost all resistant isolates, of Mycobacterium tuberculosis, ${ }^{7,9-12}$ and in a range of other bacteria. ${ }^{13,14}$

Testing M. leprae isolates for rifampicin susceptibility is generally performed after inoculation of mice ${ }^{15}$ but this is expensive and requires specialized resources that are gradually disappearing from leprosy control programmes. Radio-respirometry is a useful, more rapid alternative ${ }^{16}$ but this also needs a sophisticated laboratory infrastructure and

* Correspondence to: Prof. S. T. Cole, Unité de Génétique Moléculaire Bactérienne, Institut Pasteur, 28 Rue du Dr Roux, 75724 Paris Cedex 15, France. Phone: 33-1-45 6884 46. Fax: 33-1-40 6135 83. E-mail: stcole@pasteur.fr 
relatively large numbers of metabolically active organisms that can only be obtained by passage and growth in experimental animals. In recent years, several groups have established predictive molecular methods for detecting rifampicin resistance that are based on the $r p o B$ genotype. The first such approach involved single strand conformation polymorphism (SSCP)-PCR, ${ }^{6}$ yielding excellent results within $48 \mathrm{~h}$ and giving perfect agreement with those obtained from the mouse. Similar results were subsequently obtained using PCRfacilitated heteroduplex analysis (HDA) of $\mathrm{rpoB}^{7}$ and this method has also been applied to detecting dapsone resistance. ${ }^{17}$ However, both PCR-SSCP and PCR-HDA require gel electrophoresis and sophisticated laboratory equipment and may not be suitable for resourcepoor settings. Here we describe the development of a simpler method for predicting rifampicin resistance, involving solid-phase hybridization to oligonucleotide capture probes, and its application in the field.

\section{Materials and methods}

MEMBRANE PREPARATION

Oligonucleotides corresponding to both strands of the RIF-RDR region were designed (Figure 1, Table 1) then tested empirically for use as capture probes. The best results were obtained with probes corresponding to the complementary strand so these were then used exclusively. Capture probes were synthesized with a $5^{\prime}$-amino group to facilitate covalent linkage to Biodyne $\mathrm{C}$ membranes and diluted to the optimized concentrations in $350 \mu \mathrm{l}$ of $500 \mathrm{mM} \mathrm{NaHCO} 3, \mathrm{pH} 8.4$. Membranes $(9.5 \times 7 \mathrm{~cm})$ were activated by incubation in $20 \mathrm{ml}$ freshly prepared $16 \%$ (w/v) 1-ethyl-3-(3-dimethylaminopropyl) carbodiimide (EDAC), with shaking at room temperature for $10 \mathrm{~min}$, rinsed with water and placed in a clean miniblotter system. Diluted oligonucleotides solutions $(350 \mu \mathrm{l})$ were added to the slots and left for $5 \mathrm{~min}$ before removal. The membrane was removed from the miniblotter, inactivated in $100 \mathrm{mM}$ $\mathrm{NaOH}$ for $10 \mathrm{~min}$, then washed in $2 \times \mathrm{SSPE} / 0 \cdot 1 \%$ SDS for $5 \mathrm{~min}$ at $60^{\circ} \mathrm{C}$. After washing in $20 \mathrm{mM}$ EDTA pH 8, for $15 \mathrm{~min}$, membranes were stored at $4^{\circ} \mathrm{C}$ in Saranwrap, then cut into appropriate strips before use.

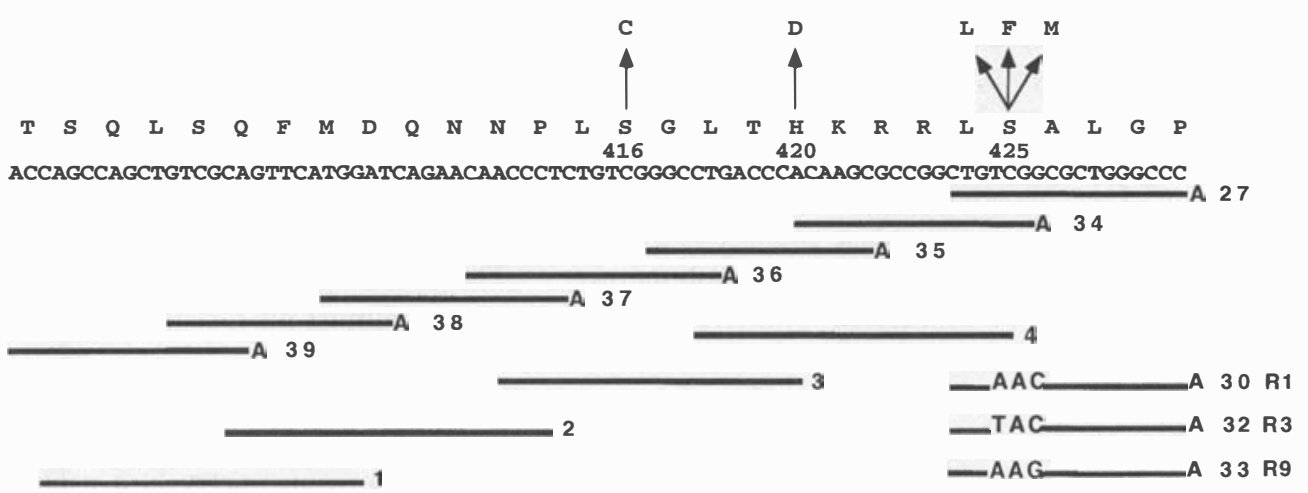

Figure 1. Organisation and sequence of the RIF-RDR of $r p o B$ from $M$. leprae and location of capture probes. The upper part shows the DNA sequence and predicted protein sequence together with mutations known or suspected to confer rifampicin resistance. The lower part shows the position of the probes discussed in the text; their sequences may be found in Table 1. 
Table 1. Oligonucleotides used in this study*

$\begin{array}{ll}\begin{array}{l}\text { For PCR and sequencing } \\ \text { Brpo22 }\end{array} & \text { bCAGGACGTCGAGGCGATCAC } \\ \text { rpo32 } & \text { TCCTCGTCAGCGGTCAAGTA } \\ \text { rpo46 } & \text { TCGATCGGGCACATCCGGC } \\ \text { Capture probes } \\ \text { S1 } \\ \text { S2 } & \text { aCCATGAACTGCGACAGCTGGCTG } \\ \text { S3 } & \text { aGGGTTGTTCTGATCCATGAACTG } \\ \text { S4 } & \text { aGACAGCCGGCGCTTGTGGGTCAG } \\ \text { A27 } & \text { aAAAGGGCCCAGCGCCGACAG } \\ \text { A30R1 } & \text { aAAAGGGCCCAGCGCCAACAG } \\ \text { A32R3 } & \text { aAAAGGGCCCAGCGCCATCAG } \\ \text { A33R9 } & \text { aAAAGGGCCCAGCGCGAACAG } \\ \text { A34 } & \text { aAAACGACAGCCGGCGCTTGT } \\ \text { A35 } & \text { aAAAGCTTGTGGGCAGGCCC } \\ \text { A36 } & \text { aAAAAGGCCCGACAGAGGGTT } \\ \text { A37 } & \text { aAAAAGGGTTGTCTGATCCA } \\ \text { A38 } & \text { aAAAGATCCATGAACTGCGAC } \\ \text { A39 } & \text { aAAATGCGACAGCTGGCTGGT }\end{array}$

a, denotes the position of the $5^{\prime}$-amino link group; $b$, denotes biotin.

PREPARATION OF BIOTINYLATED PROBES AND DNA SEQUENCING

PCR was performed using the primers B-rpo22 and rpo32 (Table 1), where B-rpo22 is biotinylated, with samples of $M$. leprae DNA prepared by the freeze-boiling method, ${ }^{18}$ as described previously. ${ }^{5}$ For control purposes, plasmids carrying cloned RIF-RDR from wild type and three rifampicin resistant isolates of $M$. leprae were used in PCR reactions. In some experiments, DNA sequencing was performed to confirm the presence of mutations predicted by the kit. Primers B-rpo22 and rpo32 were used to generate a PCR fragment spanning the RIF-RDR, which was then sequenced using primer rpoB46 (Table 1) and fluorescent big dye terminators. Sequences were obtained using an ABI373 automated sequencer.

\section{HYBRIDIZATION AND DETECTION}

This requires the following prewarmed buffers $\left(20^{\circ} \mathrm{C}\right.$, unless specified otherwise): denaturation buffer (DB) $2 \mathrm{M} \mathrm{NaOH} / 2 \mathrm{mM}$ EDTA; hybridization buffer (HB) $6 \times \mathrm{SSPE} / 0 \cdot 1 \% \mathrm{SDS}$, $50^{\circ} \mathrm{C}$; washing buffer (WB1) $4 \times \mathrm{SSPE} / 0 \cdot 1 \% \mathrm{SDS}, 55^{\circ} \mathrm{C}$, and $42^{\circ} \mathrm{C}$; washing buffer (WB2) $2 \times \mathrm{SSPE} / 0 \cdot 1 \% \mathrm{SDS}$; washing buffer (WB3) $2 \times \mathrm{SSPE}$; staining buffer (SB) $0 \cdot 1 \mathrm{M}$ Tris-buffer $\mathrm{pH} 9 \cdot 5 / 0 \cdot 05 \mathrm{M} \mathrm{MgCl}_{2}, 0 \cdot 1 \mathrm{M} \mathrm{NaCl}$. PCR fragments were denatured in an equal volume of $\mathrm{DB}$ for $5 \mathrm{~min}$ then added to prewarmed $\mathrm{HB}\left(1 \mathrm{ml}, 55^{\circ} \mathrm{C}\right)$, and incubated with the strips, in a shaking water-bath at $50^{\circ} \mathrm{C}$, for $30-60 \mathrm{~min}$. After discarding the hybridization solution and washing the strips with $2 \mathrm{ml}$ of prewarmed WB1 for $15 \mathrm{~min}$, the appropriate streptavidin-conjugate in WB1 $\left(2 \mathrm{ml}, 42^{\circ} \mathrm{C}\right)$ was added and incubated for 30-60 min. Streptavidin-peroxidase conjugate was diluted $1 / 4000$ for chemiluminescent detection and streptavidin-alkaline phosphatase conjugate was diluted 1/1000 for colorimetric detection. After discarding the conjugate solution, strips were washed with $2 \mathrm{ml}$ of WB2 for $10 \mathrm{~min}$ 
before adding $2 \mathrm{ml}$ of WB3 and washing for $10 \mathrm{~min}$. For chemiluminescent detection, strips were incubated for $2 \mathrm{~min}$ in $2 \mathrm{ml} \mathrm{ECL}$ detection liquid (Amersham), washed, then covered with Saran-wrap and exposes to X-ray film for $1 \mathrm{~min}$. For colorimetric detection, $2 \mathrm{ml}$ of diluted NBT/BCIP stock solution in SB $(200 \mu \mathrm{l} / 10 \mathrm{ml})$, was added and the strips incubated for $15-60 \mathrm{~min}$.

\section{Results}

STANDARDIZING THE METHOD

The aim of this work was to construct and standardize a kit that allows mutations to be detected in the $r p o B$ gene of $M$. leprae, and hence to predict whether the corresponding isolates would be sensitive or resistant to rifampicin. The approach involved the synthesis of a series of oligonucleotide capture probes spanning the lower strand of the RIF-RDR, their immobilization on a nylon membrane and use in reverse hybridization experiments. Many oligonucleotide probes of different lengths spanning RIF-RDR were synthesized, bound to the membrane then tested with biotinylated probes prepared from wild type and mutant $r p o B$ alleles by PCR. The optimal combination of oligonucleotides, their sequence and location is shown in Figure 1. We found that the best results were obtained by combining four 23-mer probes with six (or seven) 17-mer probes for the bottom strand. Hybridization results were uneven in initial experiments with the shorter probes, probably due to steric hindrance from the membrane resulting in less of the capture probe being available to form hybrids. Consequently, these probes were resynthesized with three additional deoxyadenosine residues at the $5^{\prime}$-end thereby allowing better access of the biotinylated PCR fragment to the 17-mer capture sequence.

To date, only a limited number of mutations responsible for rifampicin resistance have been reported in M. leprae. ${ }^{5,7}$ The amino acid substitutions involved are His420Asp (position 525 in Escherichia coli), Ser425Leu, Ser425Met and Ser425Phe. For control purposes, 17mer capture probes (with $5^{\prime}$-adenosine) corresponding to mutant $r p o B$ alleles of Ser 425 were also synthesized and included on the membranes. This proved particularly useful as the DNA sequence encompassing Ser425 is very GC-rich and readily adopts stable secondary structures that of ten preclude hybridization.

Typical results obtained with this method are shown in Figure 2. Hybridization to capture probes $1-4$ is seen in all cases except when a mutation affecting the codon for His 420 is present as probe 4 then displayed no signal (column 5). Mutations affecting this codon can also be detected with probe A35. Probe 4 also covers part of the mutational hot-spot corresponding to codon 425 but does not detect mutations affecting this position probably because the site is too close to the $5^{\prime}$-end of the capture probe for heteroduplex formation to be sufficiently destabilized. However, the shorter probe A27, in combination with the mutant probes A30R1, A32R3 and A33R9, allows all known mutations affecting codon 425 to be detected efficiently (Figure 2, columns 2, 3 and 4). The mutant probes displayed excellent specificity as no signals due to crosshybridization with the wild type $r p o B$ gene were obtained. Probes A34 and A37 sometimes displayed weaker signals than the others but the corresponding region are well covered by probes A27 and 4, or 2 and 3, respectively (Figure 1). Mutations were correctly revealed under standard laboratory conditions using either colorimetric or chemiluminescent detection methods. 


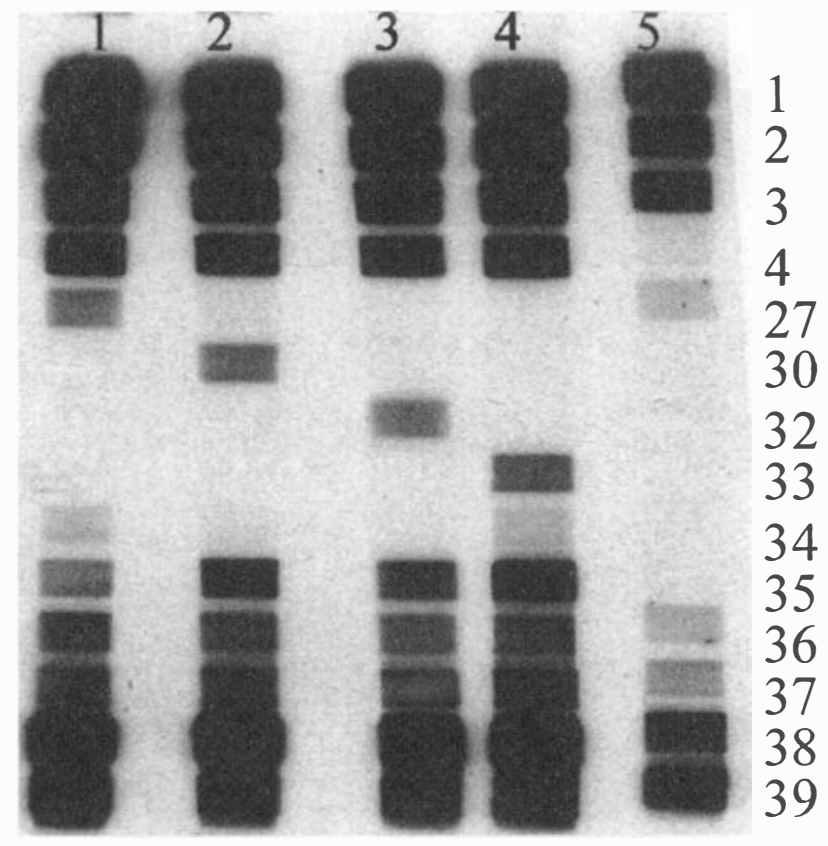

Figure 2. Detection of rifampicin resistance by reverse hybridization. The results were obtained using the protocol described in Materials and methods with biotinylated probes generated by PCR using M. leprae DNA from the following sources: column 1, wild type $M$. leprae; column 2, positive control rpoBSer425Leu; column 3, positive control rpoBSer425Met; column 4, positive control rpoBSer425Phe; column 5, strain 92041 with rpoBHis420Asp mutation. The capture probes are indicated on the right, for further details see Table 1 or Figure 1.

\section{BLIND TESTING}

Having established that the method reproducibly detected the common $r p o B$ mutations at our disposal, it was then evaluated blindly on a set of six uncharacterized $M$. leprae isolates. Five of these proved to be wild type for $\operatorname{rpoB}$ (represented in column 2, Figure 3), but one of them appeared to harbour an $r p o B$ mutation as no signal was obtained with capture probes 3 and A36 (column 1, Figure 3). These results were confirmed by DNA sequencing of PCR fragments spanning the RIF-RDR as the missense mutation Ser416Cys (TCG->TGT) was uncovered. Mutation of this residue to Leu and Ser has been reported in rifampicin resistant isolates of $M$. tuberculosis and E. coli, respectively. ${ }^{9,11,13}$

\section{FIELD TESTING}

Kits for detecting rifampicin resistance were established in Paris then tested in Kathmandu at the Anandaban Leprosy Hospital. The results obtained there were of comparable quality to those generated in France as were those obtained with membranes produced locally. A potential case of rifampicin resistance was successfully detected in a mouse footpad-derived specimen. The corresponding strain was predicted to harbour a Ser425Phe mutation by solidphase hybridization and this was confirmed by DNA sequencing. An $M$. leprae isolate harboring the corresponding allele has previously been shown to be drug resistant in the 


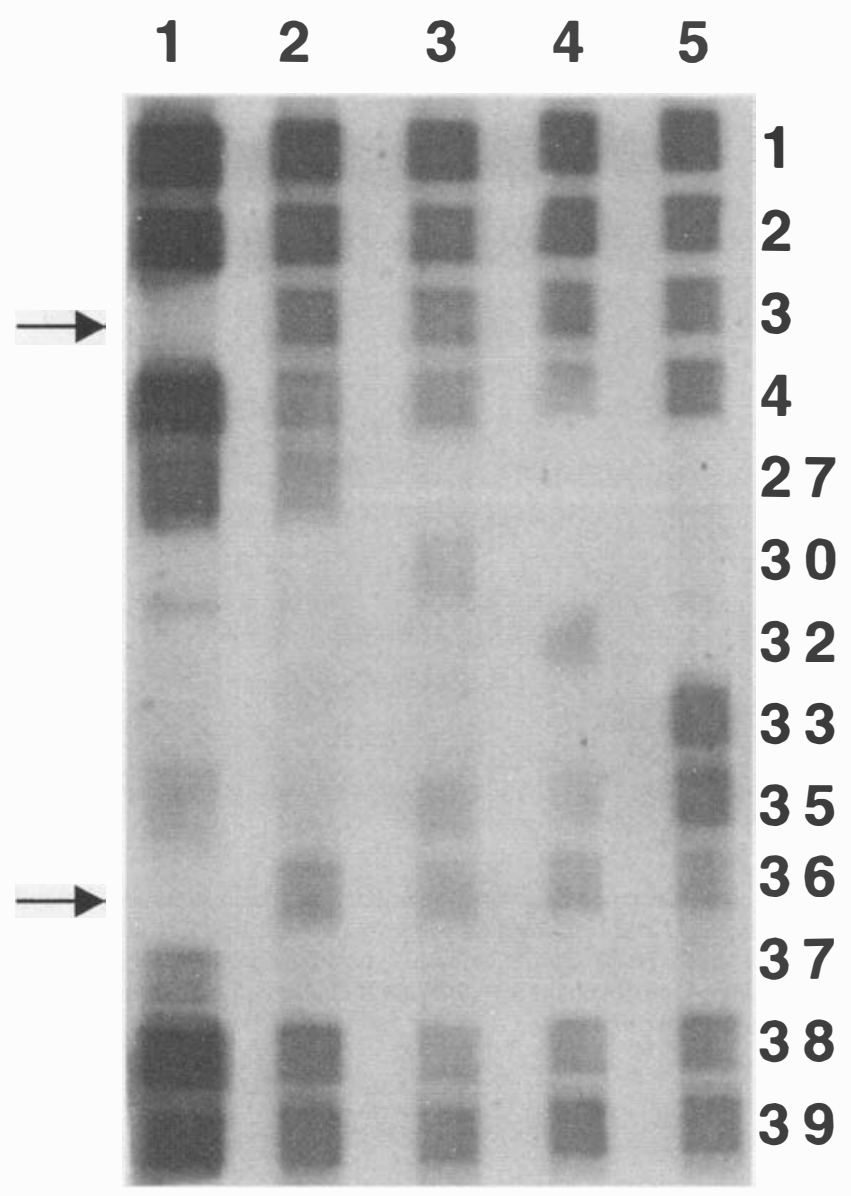

Figure 3. Detection of rifampicin resistance by reverse hybridization. As stated in legend to Figure 2 except: column 1, strain 25200 with rpoBSer416Cys mutation; column 2, wild type $M$. leprae; column 3, positive control rpoBSer425Leu; column 4, positive control rpoBSer425Met; column 5, positive control rpoBSer425Phe.

mouse model. ${ }^{4,5}$ Having validated the system, attempts were then made to apply it to clinical specimens obtained from leprosy patients.

Skin biopsies were collected from $\sim 60$ lepromatous leprosy patients in Nepal and India, stored in $70 \%$ ethanol, then transported to the Anandaban Leprosy Hospital for further analysis. Most of these patients had either relapsed or not responded to treatment. After rehydration and mincing, the biopsies were prepared for PCR analysis by freeze-boiling. Only five samples reproducibly yielded PCR fragments and these were used successfully with the chemiluminescent detection system. Two samples from patients who had not responded to treatment were found to be resistant by both methods and harboured the Ser425Phe mutation. The remaining three samples were found to be wild type, or rifampicin-susceptible, by both methods. 


\section{Discussion}

This study has convincingly demonstrated that detection of rifampicin resistance by molecular methods is a feasible and practical alternative to the mouse footpad assay. Equivalent results were obtained using two different non-radioactive detection systems in laboratories in France and Nepal. There is thus no technical reason why a kit such as that described here should not find more extensive application in developing countries. A similar method has been developed for detecting rifampicin resistant $M$. tuberculosis and is now commercially available. ${ }^{19}$ It has yielded satisfactory results in several settings. ${ }^{10}$ The availability of the $M$. leprae genome sequence also provides us with means to develop genotypic methods for detecting resistance to other drugs. This could now be done for dapsone and fluoroquinolones and, in the near future for other therapeutic agents as information about the molecular basis of resistance becomes available.

We believe that the time is now right to extend the study and to learn from the present work as this approach has much to offer leprosy control programmes. In the next phase, a much larger number of specimens should be tested. Although every available mutation has been detected by the reverse hybridization system, the number of sites within rpoB that can be monitored by positive controls is limited by the restricted set of base changes described. $^{5-7}$ As a result, the faint possibility that new mutations might be missed by the system cannot yet be excluded and more extensive evaluation is required.

This could be done in the framework of a prospective study of rifampicin resistance in which skin biopsies are examined from ex-multibacillary leprosy patients who have not responded properly to the treatment of MDT. Combined with PCR-based, DNA sequence analysis of the $r p o B$ gene from $M$. leprae extracted from the biopsies rigorous appraisal of the reverse hybridization kit would be obtained and valuable information about the level of rifampicin resistance generated. Such a study would also enable us to evaluate, in a systematic manner, the long-term efficacy of multidrug therapy for multibacillary leprosy.

\section{Acknowledgements}

We wish to acknowledge the financial support of the Institut Pasteur, the Association Française Raoul Follereau, and the UNDP/World Bank/WHO Special Programme for Research and Training in Tropical Diseases.

\section{References}

1 Grosset JH, Ji B. Recent advances in the chemotherapy of leprosy. Lepr Rev, 1990; 61: 313-329.

2 WHO Study Group. Chemotherapy of leprosy for control programmes. World Health Organisation, Geneva, Tech. Rep. Ser. 675, 1982.

3 Ji B, Grosset J. Combination of rifapentine-moxifloxacin-minocycline (PMM) for the treatment of leprosy. Lepr Rev, 2000; 71: S81-7.

${ }^{4}$ Grosset JH, Guelpa-Lauras CC, Bobin P et al. Study of 39 documented relapses of multibacillary leprosy after treatment with rifampin. Int J Lepr, 1989; 57: 607-614.

5 Honoré N, Cole ST. Molecular basis of rifampin resistance in Mycobacterium leprae. Antimicrob Agents Chemother, 1993; 37: 414-418.

6 Honoré N, Perani E, Telenti A et al. A simple and rapid technique for the detection of rifampin resistance in Mycobacterium leprae. Int J Lepr, 1994; 61: 600-604.

7 Williams DL, Waguespack C, Eisenach $\mathrm{K}$ et al. Characterization of rifampin resistance in pathogenic mycobacteria. Antimicrob Agents Chemother, 1994; 38: 2380-2386. 
${ }^{8}$ Cambau E, Perani E, Guillemin I et al. Multidrug resistance to dapsone, rifampicin, and ofloxacin in Mycobacterium leprae. Lancet, 1997; 349: 103-104.

9 Telenti A, Imboden P, Marchesi F et al. Detection of rifampicin resistance mutations in Mycobacterium tuberculosis. Lancet 1993; 341: 647-650.

10 Telenti A, Honoré N, Bernasconi C et al. Genotypic assessment of isoniazid and rifampin resistance in Mycobacterium tuberculosis: a blind study at reference laboratory level. J Clin Microbiol, 1997; 35: 719-723.

11 Ramaswamy S, Musser JM. Molecular genetic basis of antimicrobial agent resistance in Mycobacterium tuberculosis: 1998 update. Tuber Lung Dis, 1998; 79: 3-29.

12 Miller LP, Crawford JT, Shinnick TM. The rpoB gene of Mycobacterium tuberculosis. Antimicrob Agents Chemother, 1994; 38: 805-811.

13 Jin DJ, Gross CA. Mapping and sequencing of mutations in the Escherichia coli rpoB gene that lead to rifampicin resistance. J Mol Biol, 1988; 202: 45-58.

14 Carter PE, Abadi FJ, Yakubu DE, Pennington TH. Molecular characterization of rifampin resistant Neisseria meningitidis. Antimicrob Agents Chemother, 1994; 38: 1256-1261.

15 Shephard CC, Chang YT. Effect of several anti-leprosy drugs on multiplication of human leprosy bacilli in footpads of mice. Proc Soc Exp Biol Med, 1962; 109: 636-638.

16 Franzblau S. Drug susceptibility testing of Mycobacterium leprae in the BACTEC 460 system. Antimicrob Agents Chemother, 1989; 33: 2115-2117.

17 Williams DL, Pittman TL, Gillis TP et al. Simultaneous detection of Mycobacterium leprae and its susceptibility to dapsone using DNA heteroduplex analysis. J Clin Microbiol, 2001; 39: 2083-2088.

18 Woods SA, Cole ST. A rapid method for the detection of potentially viable Mycobacterium leprae in human biopsies: a novel application of PCR. FEMS Microbiol Lett, 1989; 65: 305-310.

19 Beenhouwer De H, Lhiang Z, Jannes G et al. Rapid detection of rifampicin resistance in sputum and biopsy specimens from tuberculosis patients by PCR and line probe assay. Tuberc Lung Dis, 1995; 76: 425-430. 